ARTICLE

Received 11 Apr 2013 | Accepted 1 Jul 2013 | Published 29 Jul $2013 \quad$ DOl: 10.1038/ncomms3227

\title{
Performance enhancement of fullerene-based solar cells by light processing
}

Zhe $\mathrm{Li}^{1, \star}$, Him Cheng Wong ${ }^{2, \star}$, Zhenggang Huang ${ }^{1}$, Hongliang Zhong ${ }^{1}$, Ching Hong Tan ${ }^{1}$, Wing Chung Tsoi ${ }^{3}$, Ji Seon Kim ${ }^{3}$, James R. Durrant ${ }^{1} \&$ João T. Cabral ${ }^{2}$

\begin{abstract}
A key challenge to the commercialization of organic bulk heterojunction solar cells is the achievement of morphological stability, particularly under thermal stress conditions. Here we show that a low-level light exposure processing step during fabrication of blend polymer: $\mathrm{PC}_{60} \mathrm{BM}$ solar cells can result in a 10 -fold increase in device thermal stability and, under certain conditions, enhanced device performance. The enhanced stability is linked to the light-induced oligomerization of $\mathrm{PC}_{60} \mathrm{BM}$ that effectively hinders their diffusion and crystallization in the blend. We thus suggest that light processing may be a promising, general and cost-effective strategy to optimize fullerene-based solar cell performance. The low level of light exposure required suggests not only that this may be an easily implementable strategy to enhance performance, but also that light-induced $\mathrm{PC}_{60} \mathrm{BM}$ oligomerization may have inadvertently influenced previous studies of organic solar cell device behaviour.
\end{abstract}

\footnotetext{
${ }^{1}$ Centre for Plastic Electronics and Department of Chemistry, Imperial College London, London SW7 2AZ, UK. ${ }^{2}$ Centre for Plastic Electronics and Department of Chemical Engineering, Imperial College London, London SW7 2AZ, UK. ${ }^{3}$ Centre for Plastic Electronics and Department of Physics, Imperial College London, London SW7 2AZ, UK. * These authors contributed equally to this work. Correspondence and requests for materials should be addressed to J.R.D. (email: j.durrant@imperial.ac.uk) or to J.T.C. (email: j.cabral@imperial.ac.uk)
} 
$\mathrm{R}$ ecent advances in materials and device design have led to power conversion efficiencies exceeding $9 \%$ for solutionprocessed organic solar cells (OSCs) ${ }^{1}$, approaching the benchmark required for commercial viability. However, increasing the lifetime of plastic photovoltaics remains a major challenge for their commercialization, with both light and heat shown to be stress factors that can induce device degradation ${ }^{2}$. Solution-processed OSCs are typically based on bulk heterojunction films of semiconducting polymers blended with the soluble fullerene derivative $\mathrm{PC}_{60} \mathrm{BM}\left([6,6]\right.$-phenyl- $\mathrm{C}_{61}$-butyric acid methyl ester $)^{3}$ or its larger analogue $\mathrm{PC}_{70} \mathrm{BM}^{1,4}$. The processing, nanomorphology and functional analyses of such films are generally described in terms of the thermodynamics and non-equilibrium assembly of a long-chain macromolecular donor (the polymer) with a small molecule acceptor (the fullerene). A key consideration for the processing and morphological stability of such bulk heterojunction films is therefore the potential of PCBM, as a soluble small molecule, to diffuse through and intercalate within the donor polymer domains ${ }^{5,6}$.

The high electron affinity and electron mobility of fullerenes have established them as attractive electron acceptors and transport materials for organic electronic devices. Furthermore, their optical absorption in the blue/green region of the solar spectrum, particularly for $\mathrm{C}_{70}$ derivatives, can contribute significantly to solar light harvesting. Although pristine fullerenes are relatively insoluble, and therefore only amenable to thermal evaporation, derivatized fullerenes such as PCBM can be highly soluble, allowing solution processing and considerable polymer compatibility and miscibility. This contrasts with polymeric acceptors, which typically undergo larger-scale phase segregation when blended with donor polymers, and consequently yield relatively poor solar cell efficiencies ${ }^{7}$. In blend films, fullerenes show a strong tendency to form percolated networks for electron transport, often associated with their three-dimensional symmetry. As a consequence of these advantages, fullerenes are employed almost ubiquitously as the electron acceptor in OSCs.

The performance of polymer-fullerene OSCs is strongly dependent on film morphology ${ }^{8-10}$, which is often described as a bicontinuous interpenetrating network of polymer- and fullerene-rich phases. However, numerous studies have emphasized the potential importance of intimately mixed polymerfullerene domains ${ }^{11-13}$, variations in material crystallinity ${ }^{14,15}$ and in particular, the presence of relatively pure PCBM domains, and their links to efficient device performance ${ }^{15,16}$. More detrimentally, the ability of PCBM molecules, which exhibit weak intermolecular interactions, to diffuse through the blend film is often regarded as the primary cause of thermally induced degradation of solar cell performance ${ }^{5,6}$. Under thermal annealing, PCBM molecules have been shown to diffuse within the blend to form micron-sized fullerene crystals ${ }^{5,17}$, resulting in severe degradation of device performance.

It has been previously established that pristine fullerenes, such as $\mathrm{C}_{60}$ and $\mathrm{C}_{70}{ }^{18,19}$, and derivatives, such as $\mathrm{PCBM}^{20,21}$, can undergo photochemical transformation when exposed to visible and, in particular, ultraviolet radiation. Fullerene monomers can be transformed into dimers or higher oligomers, forming covalent intermolecular $\mathrm{C}-\mathrm{C}$ bonds between fullerene monomers via a ' $2+2$ cycloaddition' mechanism ${ }^{19}$. We have recently reported that $\mathrm{C}_{60}$ fullerenes are also susceptible to undergo photochemical transformation when blended within inert polymer matrices (polystyrenes, acrylates and so on) ${ }^{22}$. Their photoactive nature, even upon modest ultraviolet or visible irradiation, is effective in suppressing film dewetting, and capable of tuning fullerene nucleation length scales during thermal annealing 22 . Neutron reflectivity indicates that the spatial distribution of fullerenes remains unchanged by light exposure ${ }^{22}$. Light processing of polymer-fullerene composites appears, therefore, as a promising route for the directed assembly of functional materials.

With these ideas in mind, we were interested in evaluating the potential impact of fullerene photoinduced oligomerisation on the design and performance of organic solar cells. Employing a benchmark polymer $/ \mathrm{PC}_{60} \mathrm{BM}$ blend in films and devices, we demonstrate herein that even modest light exposure (illumination considerably below typical operation conditions) is sufficient to drive a photo-oligomerisation of the $\mathrm{PC}_{60} \mathrm{BM}$ within the blend film which profoundly changes its morphological behaviour, thereby providing both a route to enhance OSC device performance and challenging the relevance of many previous studies of OPV morphological stability (typically conducted in the dark) to practical device operating conditions.

\section{Results}

Experimental approach. We selected a blend of $\mathrm{PC}_{60} \mathrm{BM}$ and benchmark carbazole donor polymer PCDTBT (see Fig. 1a and b for molecular structures) as a model system, and investigated both supported films and functional solar cells. $\mathrm{PC}_{60} \mathrm{BM}$ instead of $\mathrm{PC}_{70} \mathrm{BM}$ was used in blends with PCDTBT, owing to its lower fabrication cost and thus higher commercialization potential, as well as its great tendency for photo-induced crosslinking (at least for $\mathrm{C}_{60}$ relative to $\left.\mathrm{C}_{70}\right)^{23}$. Devices were fabricated in a conventional architecture without oxide interlayers, thereby avoiding the conductivity effects previously reported following light soaking of devices employing for example $\mathrm{ZnO}$ electron collection layers ${ }^{24}$. All light exposure treatments employed a standard low-intensity fluorescent lamp with illumination intensity of $\sim 10 \mathrm{~mW} \mathrm{~cm}^{-2}$ under nitrogen atmosphere except where specifically stated (see Supplementary Fig. S1 for lamp spectrum). Following irradiation, films and devices were then subjected to thermal stress so as to evaluate the impact of pre-illumination upon the morphological behaviour of the samples. Additional morphological and device studies were carried out with P3HT:PC60BM and DPP-TTT:PC60BM blends (see Supplementary Fig. S2 and S8).

Light-induced morphological stabilization. We begin our study by investigating the effect of light exposure on the morphological behaviour of PCDTBT-PC ${ }_{60} \mathrm{BM}(1: 2)$ blend films under thermal annealing, in conditions analogous and exceeding solar cell operation temperatures. Previous studies have shown that thermal annealing of polymer-fullerene blend films above a threshold temperature results in $\mathrm{PC}_{60} \mathrm{BM}$ diffusion to form micron-sized crystals visible in optical microscopy ${ }^{6,25}$. This large-scale crystallization is very detrimental to the device operation, which typically requires nm-sized phase separation ${ }^{25}$. The evolution of film morphology upon annealing above the polymer's glass transition temperature $T_{\mathrm{g}}\left(\sim 106^{\circ} \mathrm{C}\right.$ for PCDTBT), with and without light processing, is shown in Fig. 1. As-cast blend films are uniform and free of macroscopic $\mathrm{PC}_{60} \mathrm{BM}$ crystallites (Fig. 1c). Upon light exposure (in $\mathrm{N}_{2}$ atmosphere), no morphological changes are observed (data not shown). Upon thermal annealing $\left(140^{\circ} \mathrm{C}\right.$ for $1 \mathrm{~h}$ in the dark), however, the unexposed 'dark' blend film undergoes rapid nucleation and growth of large $\mathrm{PC}_{60} \mathrm{BM}$ crystallites, as shown in Fig. 1d. In particular, 'chromosome' shaped $\mathrm{PC}_{60} \mathrm{BM}$ crystallites were formed after this thermal treatment, with a number density of approximately $3500 \mathrm{~mm}^{-2}$ and an average size of $7.5 \mu \mathrm{m}$. Remarkably, the number density of such macroscopic crystallites drops drastically in light-treated films upon thermal annealing (Fig. 1e). We further note that the temperature of the samples under lamp exposure is less than $35^{\circ} \mathrm{C}$ (as measured by surface thermocouple, see Methods section), indicating that the morphological stability is not caused by heating effects during 
a

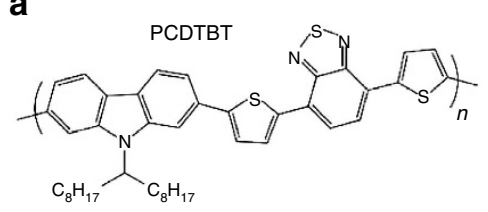

b

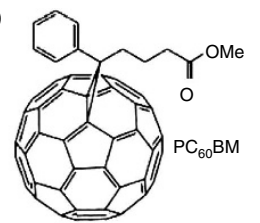

As cast
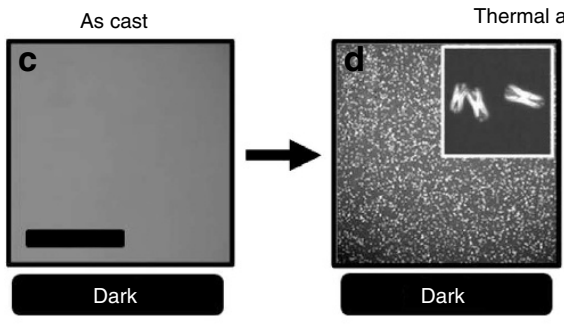

Thermal annealing $>T_{9}$
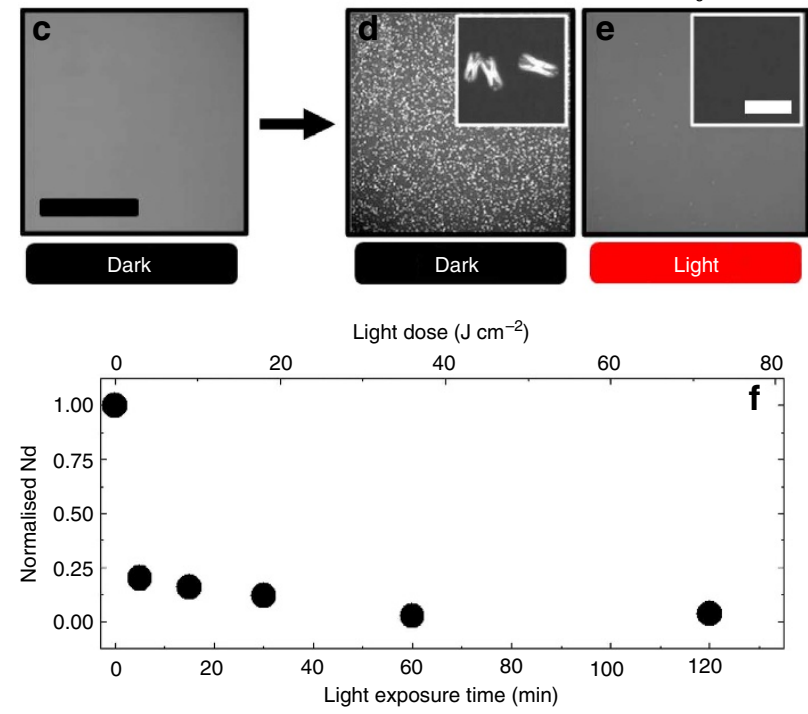

Figure 1 | Light-induced morphological stabilization. Chemical structures of (a) PCDTBT and (b) $\mathrm{PC}_{60} \mathrm{BM}$. Optical microscopy images of PCDTBT:PC ${ }_{60} B M(1: 2)$ (c) as-cast blend films (thickness $\sim 80 \mathrm{~nm}$ ) on silicon substrates (scale bar, $500 \mu \mathrm{m}$ ); (d) after thermal annealing at $140{ }^{\circ} \mathrm{C}$ for $1 \mathrm{~h}$ (dark) and (e) after thermal annealing at $140{ }^{\circ} \mathrm{C}$ for $1 \mathrm{~h}$ following fluorescent light treatment $\left(10 \mathrm{~mW} \mathrm{~cm}^{-2}, 165 \mathrm{~min}\right.$ in $\mathrm{N}_{2}$ ). The insets (scale bar, $10 \mu \mathrm{m})$ to $(\mathbf{d})$ and (e) highlight the drastic difference in the morphologies of annealed blend films without and with light exposure. (f) Shows the number density analysis of $\mathrm{PC}_{60} \mathrm{BM}$ crystals formed as a function of light dosage following thermal annealing at $140^{\circ} \mathrm{C}$ for $1 \mathrm{~h}$.

light exposure. It is thus apparent that modest light exposure of PCDTBT:PC ${ }_{60} B M$ blend films has had a profound effect on the morphological stability of the film under thermal annealing.

The suppression of $\mathrm{PC}_{60} \mathrm{BM}$ crystal formation following light exposure was also observed for other polymer- $\mathrm{C}_{60}$ and polymer$\mathrm{PC}_{60} \mathrm{BM}$ systems, including polystyrene: $\mathrm{PC}_{60} \mathrm{BM}, \mathrm{P} 3 \mathrm{HT}: \mathrm{PC}_{60} \mathrm{BM}$ and DPP-TT-T:PC ${ }_{60} \mathrm{BM}$ blends (see Supplementary Fig. S2). The suppression was observed for all thermal annealing conditions that gave rise to large-scale $\mathrm{PC}_{60} \mathrm{BM}$ crystallization, including a range of blend compositions, thermal annealing temperatures, substrates and ambient environments (nitrogen and air) (see Supplementary Fig. S3 for selected data). In all cases, low-level light exposure, for periods ranging from a few minutes to a couple of hours, was sufficient to significantly improve morphological stability of the blend films. To quantify the light dose required, we varied the light exposure times and found that $5 \mathrm{~min}$ of light exposure could be sufficient to suppress $80 \%$ of the $\mathrm{PC}_{60} \mathrm{BM}$ macroscopic crystallization upon thermal annealing, see Fig. 1f. Similarly, exposure to ambient room lights $\left(\sim 0.8 \mathrm{~mW} \mathrm{~cm}^{-2}\right)$ for $16 \mathrm{~h}$ in nitrogen was sufficient to suppress $80 \%$ of macroscopic crystallites, with this effect being accelerated in the presence of oxygen (see Supplementary Fig. S3 for selected data). This high sensitivity to light exposure suggests that the photochemical transformation driving this increase in morphological stability can be remarkably efficient. It is thus apparent that even modest inadvertent exposure to ambient lighting may have a significant impact on the morphological stability of polymer:PCBM blend films during device processing and subsequent storage/operation.

Direct evidence of $\mathbf{P C}_{60} \mathbf{B M}$ oligomerization. Previous studies have suggested that light exposure of $\mathrm{C}_{60}$ and $\mathrm{PC}_{60} \mathrm{BM}$ films can result in fullerene oligomerization, thereby substantially reducing their solubility ${ }^{18,26}$. Such fullerene oligomerization is therefore the most probable origin of the enhanced morphological stability of PCDTBT:PC ${ }_{60} \mathrm{BM}$ blend films following light exposure reported above. Direct evidence for such $\mathrm{PC}_{60} \mathrm{BM}$ oligomerization under the light exposure conditions employed in Fig. 1 is provided via gel permeation chromatography (GPC) and Raman measurements. GPC experiments were performed to detect the change in the molecular weight of the blend materials and its constituents, as the formation of new chemical bonds between $\mathrm{PC}_{60} \mathrm{BM}$ during light exposure should be reflected by the emergence of a new GPC peak corresponding to $\mathrm{PC}_{60} \mathrm{BM}$ oligomers. Measurements were done by re-dissolving the spincasted PCDTBT-PC ${ }_{60} \mathrm{BM}$ (1:2) films following $4 \mathrm{~h}$ light exposure in $\mathrm{N}_{2}$. As shown in Fig. 2a, no change was observed for the PCDTBT signal (peak i), suggesting that the PCDTBT phase was not involved in the photochemical transformation process. An extra peak (peak iii) at a molecular mass two to three times that of $\mathrm{PC}_{60} \mathrm{BM}$ (peak ii) is observed following light exposure, confirming the formation of $\mathrm{PC}_{60} \mathrm{BM}$ oligomers.

Figure $2 \mathrm{~b}$ shows the Raman spectra of pristine $\mathrm{PC}_{60} \mathrm{BM}$ and photo-oligomerized $\mathrm{PC}_{60} \mathrm{BM}$ films. The peak position of the $\mathrm{Ag}$ (2) Raman mode can be used to distinguish pristine $\mathrm{PC}_{60} \mathrm{BM}$ and $\mathrm{PC}_{60} \mathrm{BM}$ oligomers, with characteristic peak positions at 1,465 and $1,460 \mathrm{~cm}^{-1}$ respectively ${ }^{27}$. Peak analysis of the Raman signal for pristine and fluorescent light-exposed $\mathrm{PC}_{60} \mathrm{BM}$ film indicates an increased fraction of $\mathrm{PC}_{60} \mathrm{BM}$ oligomers in the latter sample (see top curve in Fig. 2b). For the pristine sample we observed a gradual shift in the peak positions of the $\mathrm{PC}_{60} \mathrm{BM} \mathrm{Ag}$ (2) mode from 1,464 to $1,461 \mathrm{~cm}^{-1}$ following exposure to the $488-\mathrm{nm}$ excitation laser (exposure time 10-110 s) indicating that the $\mathrm{PC}_{60} \mathrm{BM}$ are photo-oligomerized in situ by the 488-nm laser (see Fig. $2 \mathrm{~b}$ bottom curve).

The PCBM oligomerization reported above does not appear to have a significant impact upon its photophysical properties. Light exposure of neat $\mathrm{PC}_{60} \mathrm{BM}$ films under inert atmosphere, analogous to that employed in Figs 1 and 2, was observed to have no measurable impact on the PCBM optical absorption, and only a small redshift $(\sim 5 \mathrm{~nm})$ of the emission properties (see Supplementary Fig. S5). It thus appears that light-induced $\mathrm{PC}_{60} \mathrm{BM}$ oligomerization does not substantially affect its electronic properties, at least in terms of its highest occupied (HOMO) and lowest unoccupied molecular orbital (LUMO) energies.

Device performance enhancement by light processing. We now consider the impact of light-induced $\mathrm{PC}_{60} \mathrm{BM}$ oligomerization on the performance of benchmark PCDTBT:PC ${ }_{60} \mathrm{BM}$ OSCs. We address two issues: first the impact of this oligomerization process upon the stability of these devices under modest thermal stress; and second the potential of this oligomerization as a processing step to enhance the performance of suboptimal OSC devices. Several studies have reported that PCBM diffusion is a key factor related to the stability of OSC devices under thermal stress ${ }^{2,5,6}$. Although for P3HT: $\mathrm{PC}_{60} \mathrm{BM}$-based organic photovoltaic devices, it has been shown that limited thermal annealing can enhance performance, for devices based on most higher efficiency donor polymers, such as PCDTBT employed herein, thermal annealing typically results in degradation of the device performance. Our stability studies employed an $80^{\circ} \mathrm{C}$ dark thermal stress. Devices 
a

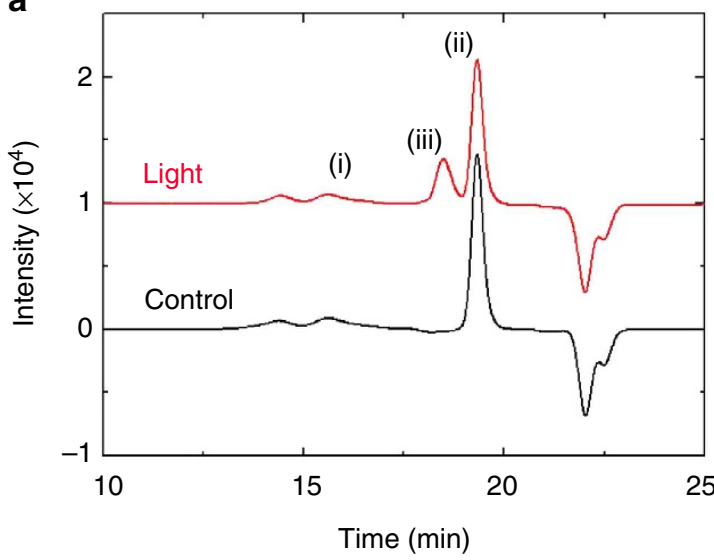

b

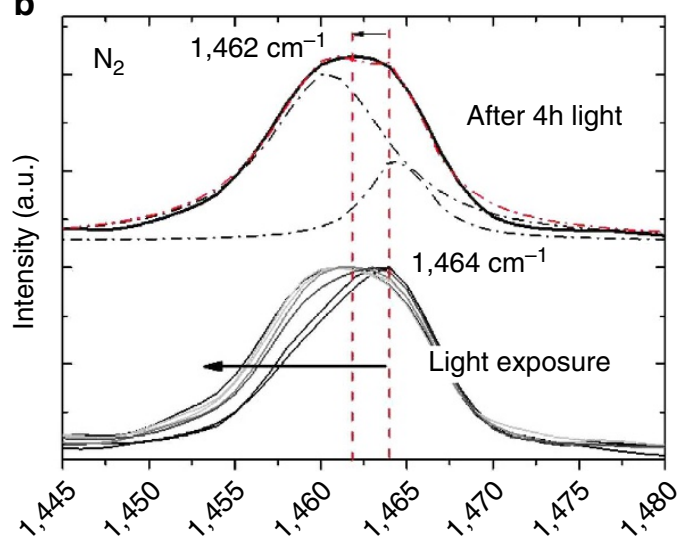

Wavenumber $\left(\mathrm{cm}^{-1}\right)$

Figure 2 | Direct evidence of $\mathbf{P C}_{\mathbf{6 0}} \mathbf{B M}$ oligomerization. (a) Gel permeation chromatography data for the PCDTBT:PC 60 BM (1:2) blend samples with and without light exposure. Control data for the individual film components (see Supplementary Fig. S4) allows us to assign these peaks to (i) PCDTBT, (ii) $\mathrm{PC}_{60} \mathrm{BM}$ and (iii) $\mathrm{PC}_{60} \mathrm{BM}$ species generated by light exposure. (b) Top: Raman data at $488 \mathrm{~nm}$ excitation wavelength (10 s acquisition time) for neat $\mathrm{PC}_{60} \mathrm{BM}$ film after being exposed to $4 \mathrm{~h}$ of fluorescent light. The data is fitted by two Lorentzian functions with peak positions at $\sim 1,460 \mathrm{~cm}^{-1}$ (oligomerized $\mathrm{PC}_{60} \mathrm{BM}$ ) and $\sim 1,465 \mathrm{~cm}^{-1}$ (pristine $\mathrm{PC}_{60} \mathrm{BM}$ ), respectively. Bottom: sequentially collected Raman spectra for initially dark $\mathrm{PC}_{60} \mathrm{BM}$ film under continuous $488 \mathrm{~nm}$ laser exposure for 10, 30, 50, 70 and $110 \mathrm{~s}$.

were fabricated in a standard architecture on ITO/PEDOT:PSS substrates, with a light exposure step $(>120 \mathrm{~min})$ introduced either before or after evaporation of a $\mathrm{Ca} / \mathrm{Al}$ top electrode. The stability of completed cells was measured using repeated dark thermal annealing/room temperature device measurement cycles. For the current-voltage $(J-V)$ measurements, the devices were exposed under a solar simulator for less than $30 \mathrm{~s}$ to minimize the effect of $\mathrm{PC}_{60} \mathrm{BM}$ oligomerization caused by this simulated solar irradiation. The samples were otherwise kept under dark conditions to eliminate any variations in experimental conditions.

Figure 3a addresses the impact of light treatment on the initial $80^{\circ} \mathrm{C}$ thermal stability of a pair of typical optimized PCDTBT: $\mathrm{PC}_{60} \mathrm{BM}$ devices (see Supplementary Fig. S6 for data averaged over five sample batches). Light treatment alone, in the absence of any heat treatment, was observed to have negligible impact on device efficiency. For the devices fabricated without light treatment, a rapid decrease in device performance was observed, with their power conversion efficiency (PCE) dropping by $10 \%$ after only $11 \mathrm{~min}$ of $80^{\circ} \mathrm{C}$ thermal annealing. This drop in PCE was primarily caused by a rapid drop in device fill factor (FF), accompanied by drops in both short circuit current $\left(J_{\mathrm{sc}}\right)$ and open circuit voltage $\left(V_{\text {oc }}\right)$ (see Supplementary Fig. S6), in agreement with previous stability studies ${ }^{28}$. In contrast, devices fabricated with light treatment exhibited significantly enhanced stability, taking $\sim 120 \mathrm{~min}$ for the PCE to drop by $10 \%$. The enhanced device stability is due to a much slower decrease in $\mathrm{FF}$, accompanied by a small increase in $V_{\text {oc }}$ during the thermal stress process. This photo-induced stability enhancement was also observed in longer timescale trials employing $20 \mathrm{~h}$ of thermal stress (see Fig. 3a). To quantify the effect of light dose on device thermal stability, we varied the light exposure time and observed a steady increase in device lifetime with increasing controlled light exposure time; see Fig. 3b. It is apparent that a somewhat longer light exposure time is required to enhance device stability compared with that required to inhibit macroscopic $\mathrm{PC}_{60} \mathrm{BM}$ crystallization in films annealed above $T_{\mathrm{g}}$ (Fig. 1d). Nevertheless, only $150 \mathrm{~min}$ of $10 \mathrm{~mW} \mathrm{~cm}^{-2}$ white light irradiation was sufficient to substantially enhance device morphological stability. Similar stability data were also obtained for light treatment before or after electrode deposition (see Supplementary Fig. S7). It is thus apparent that the $\mathrm{PC}_{60} \mathrm{BM}$ oligomerization and the associated enhanced morphological stability induced by modest light exposure during device fabrication (as observed in Figs 1 and 2) results in an approximately 10 -fold increase in the overall stability of these benchmark OSCs under thermal stress conditions.

The observed enhancement in device stability is not specific to PCDTBT:PC ${ }_{60} \mathrm{BM}$ devices; an analogous enhancement of morphological stability was also observed for devices employing the low-bandgap polymer DPP-TT-T blended with $\mathrm{PC}_{60} \mathrm{BM}$ (see Supplementary Fig. S8). These data show that the light-induced enhanced morphological stability apparent from our $\mathrm{PC}_{60} \mathrm{BM}$ crystallization studies above $T_{\mathrm{g}}$ in films also extends to enhanced photovoltaic device stability under temperature stress conditions relevant to practical device performance.

We finally consider the potential use of light treatment as a processing step to optimize and enhance device performance. Thermal annealing is widely used as a processing step to tune blend morphology and improve performance of as-cast, nonequilibrium films. Thermal annealing has been shown to be an effective processing step for P3HT:PCBM solar cells ${ }^{29}$, but has not been previously shown to be effective in enhancing the efficiency of PCDTBT:PCBM devices. Figure $3 c$ demonstrates the potential impact of light treatment upon such thermal annealing processing for PCDTBT:PC ${ }_{60} \mathrm{BM}$ devices, employing suboptimal film deposition conditions such that the initial device performance is relatively poor (PCE of $\sim 4 \%$, see Methods for experimental details). It is apparent that for a device fabricated without light treatment, thermal annealing resulted in a monotonic decrease in device performance with increasing annealing time (and annealing temperature, data not shown), consistent with previous studies ${ }^{28}$, and with Fig. $3 \mathrm{c}$. In contrast, the device fabricated with a light treatment shows a $44 \%$ increase in device performance with PCE increasing from 4.3 to $6.2 \%$ following thermal annealing at $80^{\circ} \mathrm{C}$ for $1 \mathrm{~h}$, followed by a drop in PCE with further annealing. The increase in PCE is primarily due to an increase in short circuit current $\left(J_{\mathrm{sc}}\right)$, accompanied by a mild increase in both $V_{\mathrm{oc}}$ and FF (see Supplementary Fig. S9). This drastic increase in PCE for the device with light treatment is noteworthy, and has not been observed before for PCDTBT: PCBM blends. This increase is most probably caused by the 
a

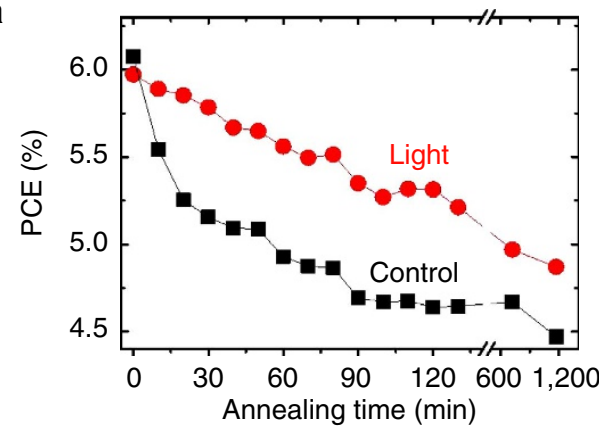

b

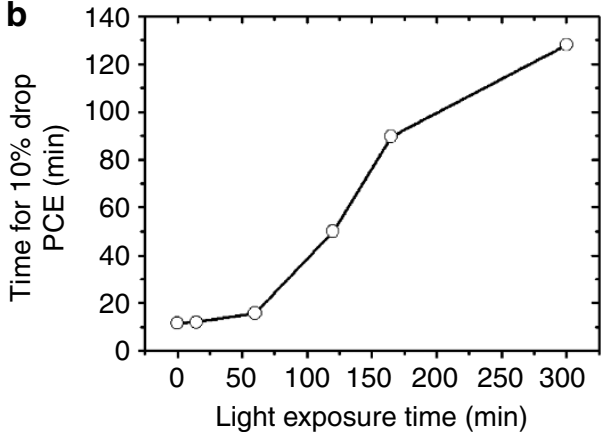

C

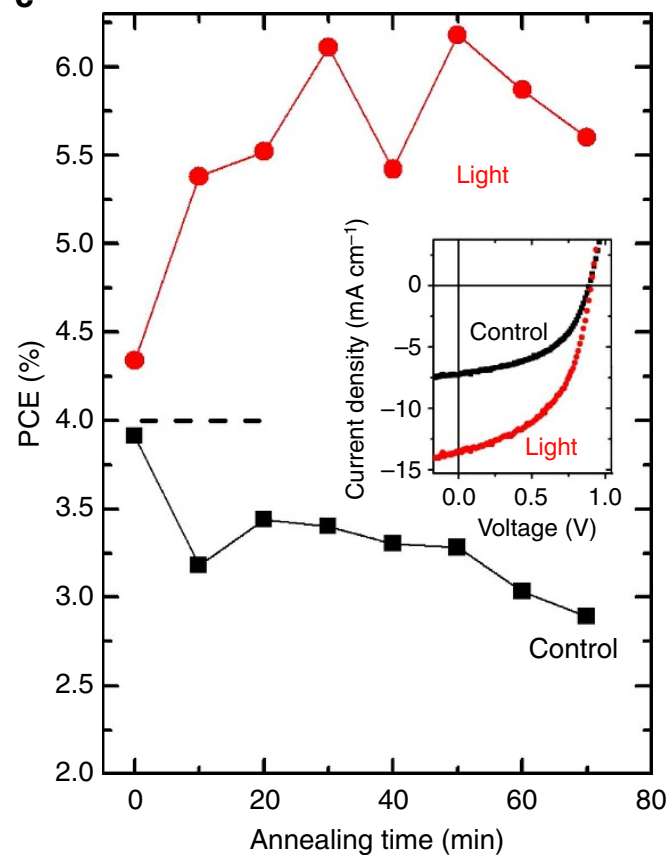

Figure 3 | Device performance enhancement by light processing. Analyses of PCDTBT:PC 60 BM solar cell PCE as a function of thermal annealing and light exposure times. PCE data in (a) and (c) are shown as a function of annealing time at $80^{\circ} \mathrm{C}$ following a fixed light exposure (2.75 h of white light $10 \mathrm{~mW} \mathrm{~cm}^{-2}$ ) as well as control data without light exposure. Plot (a) shows data for devices fabricated with optimized film deposition, whereas (c) shows data for devices with non-optimized spin-coating conditions (and therefore lower initial efficiencies). The inset to plot (c) compares the $J-V$ characteristics of the non-optimized devices in (c) with and without light exposure followed by thermal annealing at $80^{\circ} \mathrm{C}$ for 50 min. Plot (b) shows the annealing time required for a 10\% PCE loss of initially optimized devices as a function of light exposure time. Further details of device performance characteristics and data reproducibility are given in Supplementary Fig. S6.

oligomerization of $\mathrm{PC}_{60} \mathrm{BM}$ preventing the diffusion of $\mathrm{PC}_{60} \mathrm{BM}$, whereas thermal annealing enhances the ordering of the polymer phase $^{30}$. A more optimized blend morphology is also consistent with an increased $J_{\mathrm{sc}}$ and $\mathrm{FF}$ due to better charge generation and collection.

\section{Discussion}

Thermally induced morphological degradation is a key consideration for commercial application of organic bulk heterojunction solar cells. In this paper, we have reported that modest light irradiation of a benchmark polymer-fullerene blend can result in substantially enhanced morphological stability under subsequent thermal stress for both films and complete solar cells. Evidence is presented linking this enhanced stability with photo-induced $\mathrm{PC}_{60} \mathrm{BM}$ oligomerization. The magnitude of the stabilization is observed to depend on the details of film composition, annealing temperature, substrate and so on. Nevertheless, it is striking that under the conditions studied only a few minutes of modest irradiation $(\sim 1 / 10$ th of standard solar irradiation intensity) was sufficient to suppress almost completely thermally induced $\mathrm{PC}_{60} \mathrm{BM}$ macroscopic crystallization in a benchmark polymer: $\mathrm{PC}_{60} \mathrm{BM}$ film, and that $100 \mathrm{~min}$ of such modest irradiation was sufficient to increase device longevity under subsequent thermal stress by an order of magnitude.

It should be noted that recent studies on the stability of polymer-fullerene solar cells under thermal stress have reported substantial variations in performance (with, for example, times for $10 \%$ loss of PCE under thermal stress ranging from tens of minutes to $\gtrsim 100 \mathrm{~h}$ (refs $31-34)$ ). The origins of these variations are not fully resolved, but most probably related to details of device fabrication (for example, photoactive layer and electrode composition) and experimental measurement conditions (for example, light exposure, temperature, pre-evaluation initial device annealing). For example, our own studies have indicated that the longer lifetimes reported in Bertho et al. ${ }^{32}$ are most probably related to the use a pre-evaluation annealing step, so excluding any initial performance degradation from their study, and due to the use of $\mathrm{PC}_{70} \mathrm{BM}$ as an electron acceptor (which is reported to have superior morphological stability in blend films than $\left.\mathrm{PC}_{60} \mathrm{BM}^{35,36}\right)$. The stability data we report herein is clearly limited in scope and timescale, but unequivocally demonstrates that light exposure can have a positive impact upon thermal stability. For long-term stability enhancement, it is likely that continuous irradiation during thermal stress will be necessary to maintain PCBM oligomerization (prolonged thermal annealing has been shown to reverse photo-induced $\mathrm{C}_{60}$ cross-linking ${ }^{18}$ ). Such combined light and thermal stress is of particular relevance to practical solar cell operating conditions (continuous irradiation is likely to be particularly important for devices employing $\mathrm{PC}_{70} \mathrm{BM}$ as previous studies have indicated $\mathrm{C}_{70}$ is less susceptible to photo-induced crosslinking than $\mathrm{C}_{60}$ (ref. 23). Indeed it appears possible that the impressive device lifetimes reported for analogous PCDTBT:PCBM devices under solar irradiation (projected lifetimes of $\sim 6$ years $^{37}$ ) may in part be related to photo-induced PCBM oligomerization ensuring a stable film morphology.

The main conclusions of the paper are summarized graphically in Fig. 4; an additional 'light annealing' step during fullerenebased OSC fabrication can significantly improve device lifetime and optimize performance. The light exposure causes oligomerization of $\mathrm{PC}_{60} \mathrm{BM}$, which then imparts greater film morphological stability under subsequent heat stress or thermal annealing. This effect is robust to all polymer matrices and temperatures studied. We show that even modest illumination, commensurate with typical laboratory conditions, is sufficient to initiate this effect 


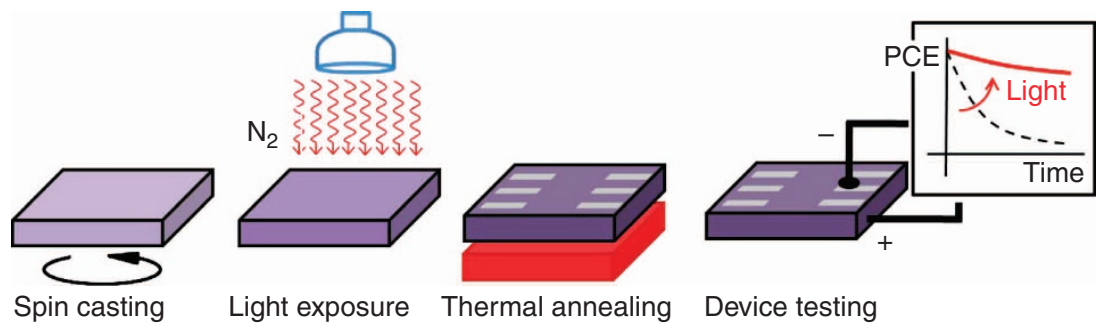

Figure 4 | Proposed experimental procedure for OSC device fabrication and stability studies. An additional controlled low-level 'light annealing' step in OSC device fabrication results in significantly enhanced device thermal stability and/or enhanced device performance.

and must therefore be monitored and controlled to ensure consistent device performance. As such, inadvertent light exposure is likely to have significantly impacted many previous studies of OSC morphological behaviour employing acceptor fullerenes. Several studies have reported the synthesis of new active layer materials that enable photo-induced polymerization or crosslinking approaches to enhance OSC device stability ${ }^{38,39}$. However, these approaches typically require increases in materials complexity and/or a loss of device efficiency. The findings reported herein suggest that photo-induced oligimerization may be readily achieved with materials already employed in optimized devices, with negligible loss of performance, thereby providing an attractive route to enhancing the commercial potential of OSCs.

\section{Methods}

Materials. PCDTBT was supplied by 1-materials and purified at Imperial with a molecular weight $\left(M_{\mathrm{w}}\right)$ of $21.6 \mathrm{~kg} \mathrm{~mol}^{-1}$ and polydispersity of $5.5 . \mathrm{PC}_{60} \mathrm{BM}$ was supplied by Nano-C. The midpoint $T_{\mathrm{g}}$ of neat PCDTBT was measured as $106 \pm 1{ }^{\circ} \mathrm{C}$ using differential scanning calorimetry (DSC TA Instruments Q2000) at the rate of $10^{\circ} \mathrm{C} \mathrm{min}^{-1}$.

Morphological stability studies. Silicon substrates (Compart Technology) were cleaned with $\mathrm{N}_{2}$ before use. Samples were made by spin-casting the active bulk heterojunction layer comprising weight ratio 1:2 PCDTBT:PC ${ }_{60} \mathrm{BM}$ on to silicon substrates with a thickness of $80 \mathrm{~nm}$. A fluorescent lamp was used as the light source, with light intensity and spectrum measured by a StellarNet EPP2000 spectrometer. Blend film morphology was observed by a reflection optical microscope (Olympus BX 41M), equipped with an XY stage and CCD camera (AVT Marlin)

Gel permeation chromatography. GPC measurements were performed using Agilent Technologies 1,200 series GPC running in chlorobenzene at $80^{\circ} \mathrm{C}$, using two PL mixed B columns in series, and calibrated against narrow polydispersity polystyrene standards.

Raman spectroscopy. Raman measurements were performed using a Renishaw In-Via Raman microscope in a back-scattering configuration on $\mathrm{PC}_{60} \mathrm{BM}$ neat and blend samples; the latter spectra are dominated by the polymer signal, which does not change upon illumination. The excitation wavelength is $488 \mathrm{~nm}$ with a power of $\sim 0.9 \mathrm{~mW}$ and a beam diameter of $\sim 10 \mu \mathrm{m}$ on the films. All the measurements were done in nitrogen environment to reduce the competing fullerene photooxidation process. In all cases, the spectra were obtained with data acquisition time of $10 \mathrm{~s}$, unless specified. The Raman spectra were background corrected using the Renishaw Wire 3.1 program. The spectral resolution is $\sim 1 \mathrm{~cm}^{-1}$.

Device fabrication and stability study. ITO glass substrates were cleaned by sonication in deionized water, acetone and isopropyl alcohol (IPA) successively, followed by an oxygen plasma treatment at $100 \mathrm{~W}$ for $7 \mathrm{~min}$ before a $35-\mathrm{nm}$-thick PEDOT:PSS layer was deposited on to the substrates and then annealed at $150{ }^{\circ} \mathrm{C}$ for $20 \mathrm{~min}$. The substrates were then transferred to $\mathrm{N}_{2}$ glovebox for deposition of the active layer. A number of device fabrication recipes were employed varying the dissolving solvent, weight ratio, solution concentration, dissolving period, spin acceleration and spin speed. For the PCDTBT:PC ${ }_{60} \mathrm{BM}$ devices fabricated in Fig. 3a, PCDTBT and $\mathrm{PC}_{60} \mathrm{BM}$ were codissolved in anhydrous chlorobenzene with a total solution concentration of $25 \mathrm{mg} \mathrm{ml}^{-1}$ and a weight ratio of 1:2. For the PCDTBT:PC ${ }_{60} \mathrm{BM}$ devices fabricated in Fig. $3 \mathrm{c}$, PCDTBT and $\mathrm{PC}_{60} \mathrm{BM}$ were codissolved in anhydrous dichlorobenzene with a total solution concentration of $40 \mathrm{mg} \mathrm{ml}^{-1}$ and a weight ratio of 1:2. Solutions were stirred in $\mathrm{N}_{2}$ glovebox at $55^{\circ} \mathrm{C}$ for more than $24 \mathrm{~h}$ followed by filtering through a $0.2-\mu \mathrm{m}$ filter. Active layer film thickness is $\sim 85-\mathrm{nm}$ thick for the devices fabricated in Fig. $3 \mathrm{a}$ and $\sim 80-\mathrm{nm}$ thick for the devices fabricated in Fig. 3c. All devices were completed by evaporation of $25 \mathrm{~nm}$ of calcium and $100 \mathrm{~nm}$ of aluminium through a 6-pixel mask. The pixel size, defined by the spatial overlap of the ITO anode and $\mathrm{Ca} / \mathrm{Al}$ cathode, was $0.045 \mathrm{~cm}^{2}$. Film thickness was determined with a Dektak $6 \mathrm{M}$ profilometer. Annealing steps in both morphology and device stability studies were carried out in a $\mathrm{N}_{2}$-filled glovebox. For device degradation studies, the same lamp was used for light exposure as the morphological studies. Annealing temperature was measured and calibrated using a Kane-May KM330 temperature sensor. The film surface temperature under illumination was measured with a surface thermocouple connected to the films on silicon or ITO/PEDOT:PSS substrates. $J-V$ characteristics were measured using a Xenon lamp at AM1.5 solar illumination (Oriel Instruments) calibrated to a silicon reference cell with a Keithley 2,400 source meter.

\section{References}

1. He, Z. et al. Enhanced power-conversion efficiency in polymer solar cells using an inverted device structure. Nat. Photon 6, 591-595 (2012).

2. Jørgensen, M. et al. Stability of polymer solar cells. Adv. Mater. 24, 580-612 (2012).

3. Dennler, G., Scharber, M. C. \& Brabec, C. J. Polymer-fullerene bulkheterojunction solar cells. Adv. Mater. 21, 1323-1338 (2009).

4. Peet, J. et al. Efficiency enhancement in low-bandgap polymer solar cells by processing with alkane dithiols. Nat. Mater 6, 497-500 (2007).

5. Yang, X. et al. Morphology and thermal stability of the active layer in poly(pphenylenevinylene)/methanofullerene plastic photovoltaic devices. Macromolecules 37, 2151-2158 (2004).

6. Ebadian, S. et al. Effects of annealing and degradation on regioregular polythiophene-based bulk heterojunction organic photovoltaic devices. Sol. Energy Mater. Sol. Cells 94, 2258-2264 (2010).

7. McNeill, C. R. \& Greenham, N. C. Conjugated-polymer blends for optoelectronics. Adv. Mater. 21, 3840-3850 (2009).

8. Salleo, A., Kline, R. J., DeLongchamp, D. M. \& Chabinyc, M. L. Microstructura characterization and charge transport in thin films of conjugated polymers. Adv. Mater. 22, 3812-3838 (2010).

9. DeLongchamp, D. M., Kline, R. J. \& Herzing, A. Nanoscale structure measurements for polymer-fullerene photovoltaics. Energy Environ. Sci. 5, 5980-5993 (2012).

10. Collins, B. A., Tumbleston, J. R. \& Ade, H. Miscibility, crystallinity, and phase development in P3HT/PCBM solar cells: toward an enlightened understanding of device morphology and stability. J. Phys. Chem. Lett. 2, 3135-3145 (2011).

11. Shoaee, S. et al. Charge photogeneration for a series of thiazolo-thiazole donor polymers blended with the fullerene electron acceptors PCBM and ICBA. Adv. Funct. Mater 23, 3286-3298 (2013).

12. Yin, W. \& Dadmun, M. A new model for the morphology of P3HT/PCBM organic photovoltaics from small-angle neutron scattering: rivers and streams. ACS Nano 5, 4756-4768 (2011).

13. Pfannmöller, M. et al. Visualizing a homogeneous blend in bulk heterojunction polymer solar cells by analytical electron microscopy. Nano. Lett. 11, 3099-3107 (2011).

14. Wu, W.-R. et al. Competition between fullerene aggregation and poly(3hexylthiophene) crystallization upon annealing of bulk heterojunction solar cells. ACS Nano 5, 6233-6243 (2011).

15. Jamieson, F. C. et al. Fullerene crystallisation as a key driver of charge separation in polymer/fullerene bulk heterojunction solar cells. Chem. Sci. 3, 485-492 (2012).

16. Kim, J. Y. \& Frisbie, C. D. Correlation of phase behavior and charge transport in conjugated polymer/fullerene blends. J. Phys. Chem. C 112, 17726-17736 (2008).

17. Bertho, S. et al. Effect of temperature on the morphological and photovoltaic stability of bulk heterojunction polymer:fullerene solar cells. Sol. Energy Mater. Sol. Cells 92, 753-760 (2008).

18. Eklund, P. C. et al. Photochemical transformation of $\mathrm{C}_{60}$ and $\mathrm{C}_{70}$ films. Thin Solid Films 257, 185-203 (1995). 
19. Rao, A. M. et al. Photoinduced polymerization of solid $\mathrm{C}_{60}$ films. Science 259, 955-957 (1993).

20. Wang, J., Larsen, C., Wagberg, T. \& Edman, L. Direct UV patterning of electronically active fullerene films. Adv. Funct. Mater. 21, 3723-3728 (2011).

21. Dzwilewski, A., Matyba, P. \& Edman, L. Facile fabrication of efficient organic CMOS circuits. J. Phys. Chem. B 114, 135-140 (2009).

22. Wong, H. C. et al. Patterning polymer-fullerene nanocomposite thin films with light. Adv. Mater. 25, 985-991 (2013).

23. Rao, A. M. et al. Photoinduced polymerization of solid $\mathrm{C}_{70}$ films. Chem. Phys. Lett. 224, 106-112 (1994).

24. Lilliedal, M. R. et al. The effect of post-processing treatments on inflection points in current-voltage curves of roll-to-roll processed polymer photovoltaics. Sol. Energy Mater. Sol. Cells 94, 2018-2031 (2010).

25. Wang, T. et al. Correlating structure with function in thermally annealed PCDTBT:PC70BM photovoltaic blends. Adv. Funct. Mater 22, 1399-1408 (2012).

26. Wang, J., Enevold, J. \& Edman, L. Photochemical transformation of fullerenes. Adv. Funct. Mater 23, 3220-3225 (2013).

27. Dzwilewski, A., Wagberg, T. \& Edman, L. Photo-induced and resist-free imprint patterning of fullerene materials for use in functional electronics. J. Am. Chem. Soc. 131, 4006-4011 (2009).

28. Abhay Gusain, V. S., Veerender, P., Jha, P., Koiry, S. P., Chauhan, A. K. \& Aswal, D. K. S.K.G. investigation on the effects of thermal annealing on PCDTBT:PCBM bulk-heterojunction polymer solar cells. AIP Conf. Proc. 1512, 776-777 (2012)

29. Ma, W. et al. Thermally stable, efficient polymer solar cells with nanoscale control of the interpenetrating network morphology. Adv. Funct. Mater. 15, 1617-1622 (2005)

30. Lu, X. et al. Bilayer order in a polycarbazole-conjugated polymer. Nat Commun. 3, 795 (2012)

31. De Bettignies, R., Leroy, J., Firon, M. \& Sentein, C. Accelerated lifetime measurements of P3HT:PCBM solar cells. Synth. Met. 156, 510-513 (2006).

32. Bertho, S. et al. Influence of thermal ageing on the stability of polymer bulk heterojunction solar cells. Sol. Energy Mater. Sol. Cells 91, 385-389 (2007).

33. Wang, D. H. et al. Stability comparison: A PCDTBT/PC ${ }_{71} \mathrm{BM}$ bulkheterojunction versus a $\mathrm{P} 3 \mathrm{HT} / \mathrm{PC}_{71} \mathrm{BM}$ bulk-heterojunction. Sol. Energy Mater. Sol. Cells 101, 249-255 (2012).

34. Ouhib, F. et al. Thermally stable bulk heterojunction solar cells based on crosslinkable acrylate-functionalized polythiophene diblock copolymers. Macromolecules 46, 785-795 (2013).
35. Labram, J. G., Kirkpatrick, J., Bradley, D. D. C. \& Anthopoulos, T. D. Impact of fullerene molecular weight on P3HT:PCBM microstructure studied using organic thin-film transistors. Adv. Energy Mater. 1, 1176-1183 (2011).

36. Boland, P. et al. Investigation of structural, optical, and electrical properties of regioregular poly(3-hexylthiophene)/fullerene blend nanocomposites for organic solar cells. Thin Solid Films 518, 1728-1731 (2010).

37. Peters, C. H. et al. High efficiency polymer solar cells with long operating lifetimes. Adv. Energy Mater. 1, 491-494 (2011).

38. Drees, M. et al. Stabilization of the nanomorphology of polymer-fullerene 'bulk heterojunction' blends using a novel polymerizable fullerene derivative. $J$. Mater. Chem. 15, 5158-5163 (2005).

39. Cheng, Y.-J., Hsieh, C.-H., Li, P.-J. \& Hsu, C.-S. Morphological stabilization by in situ polymerization of fullerene derivatives leading to efficient, thermally stable organic photovoltaics. Adv. Funct. Mater. 21, 1723-1732 (2011).

\section{Acknowledgements}

We thank Solvay SA and the EPSRC EP/J500021/1, EP/J500239/1 and EP/H040218/1 for financial support. We also thank Iain McCulloch and Hugo Bronstein for DPP-TT-T synthesis, Pabitra Shakya for their assistance with device fabrication, and Martin Heeney for access to the GPC measurements.

\section{Author contributions}

Optical microscopy measurements were performed by H.C.W. Devices were fabricated by Z.L. and Z.H. Morphology and device thermal stability studies were performed by Z.L., C.H.T. and H.C.W. GPC measurements were performed by H.Z. Raman measurements were performed by W.C.T. and J.S.K. Data analysis was performed by Z.L. and H.C.W. J.R.D. and J.T.C. contributed to project planning, experimental design and manuscript preparation.

\section{Additional information}

Supplementary Information accompanies this paper at http://www.nature.com/ naturecommunications

Competing financial interests: The authors declare no competing financial interests.

Reprints and permission information is available online at http://npg.nature.com/ reprintsandpermissions/

How to cite this article: Li, Z. et al. Performance enhancement of fullerene-based solar cells by light processing. Nat. Commun. 4:2227 doi: 10.1038/ncomms3227 (2013). 